\title{
Collaborative action of surface chemistry and topography in the regulation of mesenchymal and epithelial markers and the shape of cancer cells
}

\author{
Junsheng Li, ${ }^{1,2,6}$ Barbara Kwiatkowska, ${ }^{1}$ Hao Lu ${ }^{2}$, Maren Voglstätter ${ }^{4}$, Erica Ueda ${ }^{1}$, Michael Grunze, ${ }^{2,3}$ \\ Jonathan Sleeman ${ }^{1,4}$, Pavel A. Levkin ${ }^{1,2, *}$ and Irina Nazarenko ${ }^{1,5^{* *}}$.
}

${ }^{1}$ Institute of Toxicology and Genetics, Karlsruhe Institute of Technology, 76021 Karlsruhe, Germany

${ }^{2}$ Applied Physical Chemistry, University of Heidelberg, 69120 Heidelberg, Germany

${ }^{3}$ Institute of Functional Interfaces (IFG), Karlsruhe Institute of Technology (KIT), Germany

${ }^{4}$ University Medicine Mannheim and University of Heidelberg, Centre for Biomedicine and Medical Technology Mannheim (CBTM), D-68167 Mannheim, Germany

${ }^{5}$ Institute of Environmental Health Sciences and Hospital Infection Control, Medical Center, University of Freiburg, 79106 Freiburg, Germany

${ }^{6}$ School of Chemistry, Chemical Engineering and Life Sciences, Wuhan University of Technology, 430070, China 
(A)

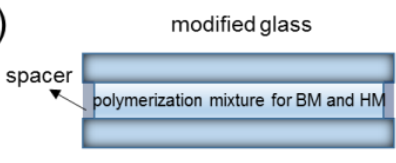

modified glass

(B)
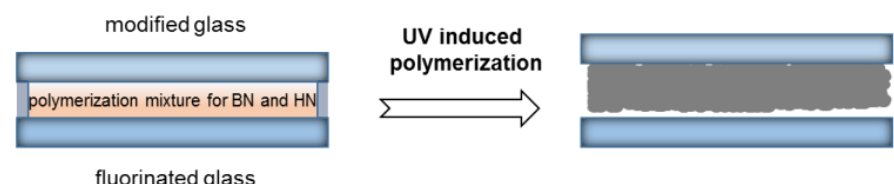

separate the glass plates

microporous surface

glass plates

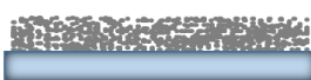

modified glass (upper one)

nanoporous/non-porous surface

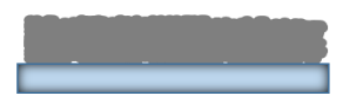

modified glass
(C)

F

i

(D)

$\mathrm{g}$

u

r

$$
\mathrm{CH}_{2} \triangleq \mathrm{H}_{\mathrm{C}}^{\mathrm{CH}_{3}} \mathrm{~S}_{\mathrm{O}}^{\mathrm{II}}-\mathrm{OH}
$$

AMPS

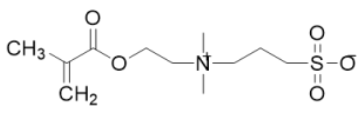

MDSA

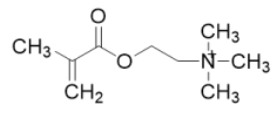

META

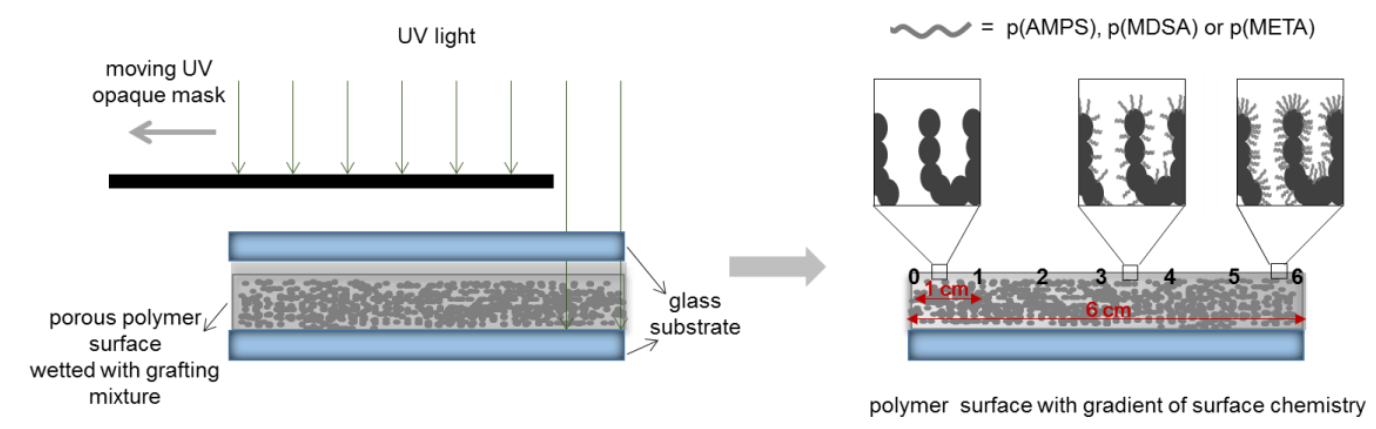

Figure S1. Schematic representation of the preparation of (A) micro-porous surface and (B) nano-porous / non-porous surfaces. (C) Acrylate and methacrylate monomers used for the surface modification via photografting. AMPS: 2-acrylamido-2-methyl-1-propanesulfonic acid; MDSA: [2-(methacryloyloxy)ethyl]dimethyl-(3-sulfopropyl)ammonium hydroxide; META: [2-(methacryloyloxy)ethyl] trimethylammonium chloride. (D) Schematic representation of the method for producing the polymer surface with gradient density of functional groups via photoinitiated grafting (left) and the resultant surface with a gradient in the grafting density (right). The properties of the prepared surface were characterized on 7 representative points (point 0 to point 7 in the scheme) of the surface and the distance between the neighboring points is $1 \mathrm{~cm}$. 

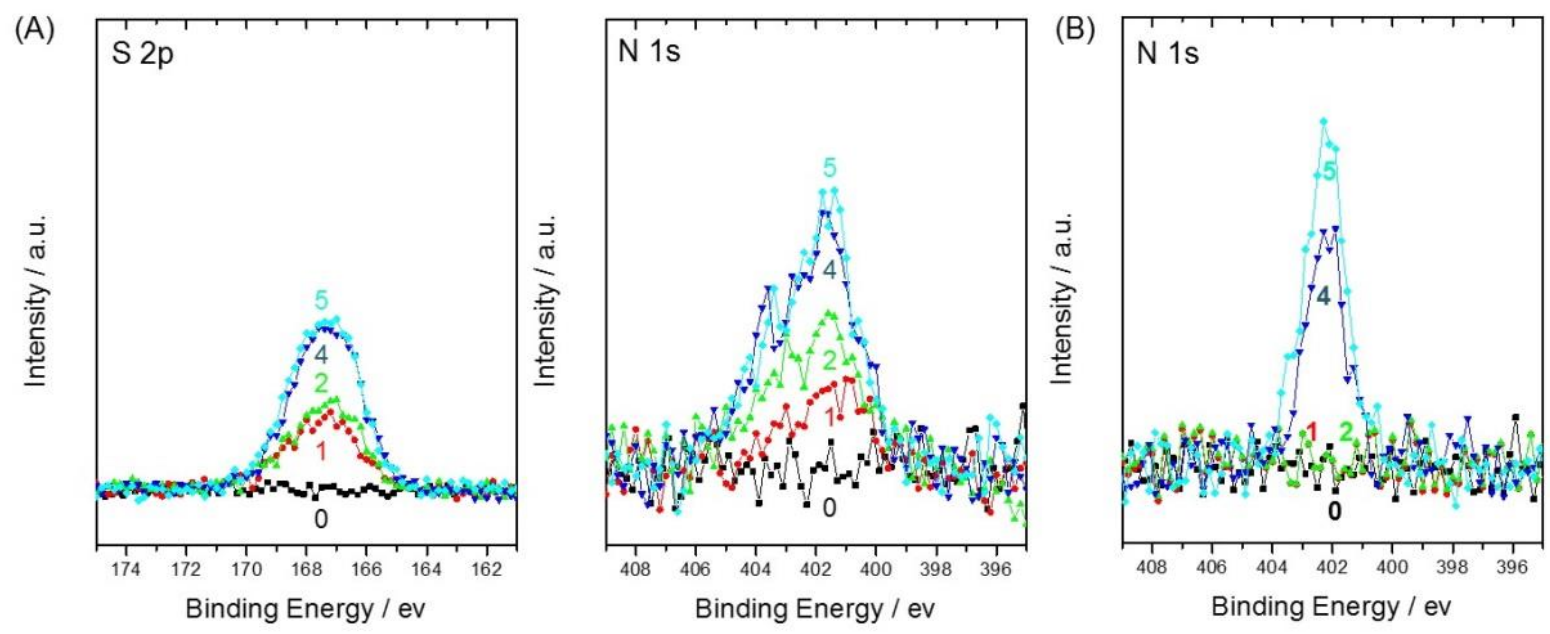

Figure S2. X-ray photoelectron spectroscopy (XPS) spectra measured at different measuring points along the surface chemistry gradient, designated as position 0, 1, 2, 4 and 5 (see Fig. 1) of the (A) BN-AMPS surface (left: S 2p spectra, right: N 1s spectra) and (B) BN-META surface.

Table S1. Atomic ratio of the element along the gradient direction of BN-AMPS, BN-MDSA and BN-META surface.

\begin{tabular}{lcccccc}
\hline \multicolumn{1}{c}{ surface } & element & position 0 & position 1 & position 2 & position 4 & position 5 \\
\hline \multirow{2}{*}{ BN-AMP } & $\mathrm{C}$ & $81.0 \%$ & $80.1 \%$ & $79.0 \%$ & $78.2 \%$ & $77.3 \%$ \\
$\mathrm{~S}$ & $\mathrm{O}$ & $19.0 \%$ & $18.2 \%$ & $18.3 \%$ & $17.2 \%$ & $17.8 \%$ \\
& $\mathrm{~N}$ & 0 & $0.8 \%$ & $1.6 \%$ & $2.5 \%$ & $2.6 \%$ \\
& $\mathrm{~S}$ & 0 & $0.9 \%$ & $1.1 \%$ & $2.1 \%$ & $2.3 \%$ \\
\hline \multirow{2}{*}{$\mathrm{BN}-\mathrm{MDS}$} & $\mathrm{C}$ & $82.2 \%$ & $79.3 \%$ & $78.4 \%$ & $74.3 \%$ & $74.9 \%$ \\
$\mathrm{~A}$ & $\mathrm{O}$ & $17.8 \%$ & $19.3 \%$ & $18.4 \%$ & $20.7 \%$ & $21.0 \%$ \\
& $\mathrm{~N}$ & 0 & $0.6 \%$ & $1.6 \%$ & $2.3 \%$ & $1.8 \%$ \\
& $\mathrm{~S}$ & 0 & $0.6 \%$ & $1.6 \%$ & $2.7 \%$ & $2.3 \%$ \\
$\mathrm{BN}-\mathrm{CET}$ & $\mathrm{C}$ & $80.7 \%$ & $81.5 \%$ & $81.6 \%$ & $81.5 \%$ & $81.0 \%$ \\
$\mathrm{~A}$ & $\mathrm{O}$ & $19.3 \%$ & $18.5 \%$ & $18.4 \%$ & $17.1 \%$ & $17.2 \%$ \\
& $\mathrm{~N}$ & 0 & 0 & 0 & $1.4 \%$ & $1.8 \%$ \\
\hline
\end{tabular}


(A)

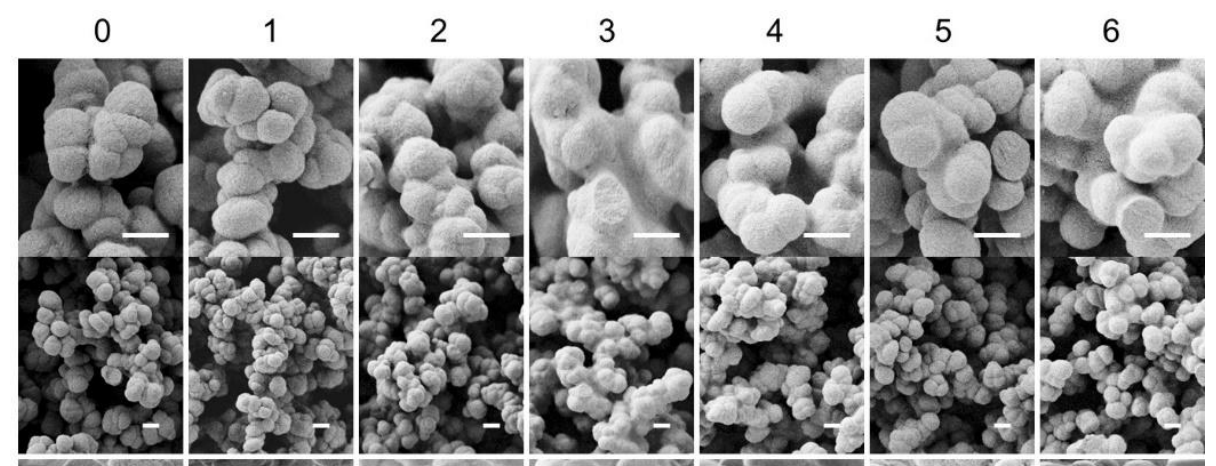

(B)

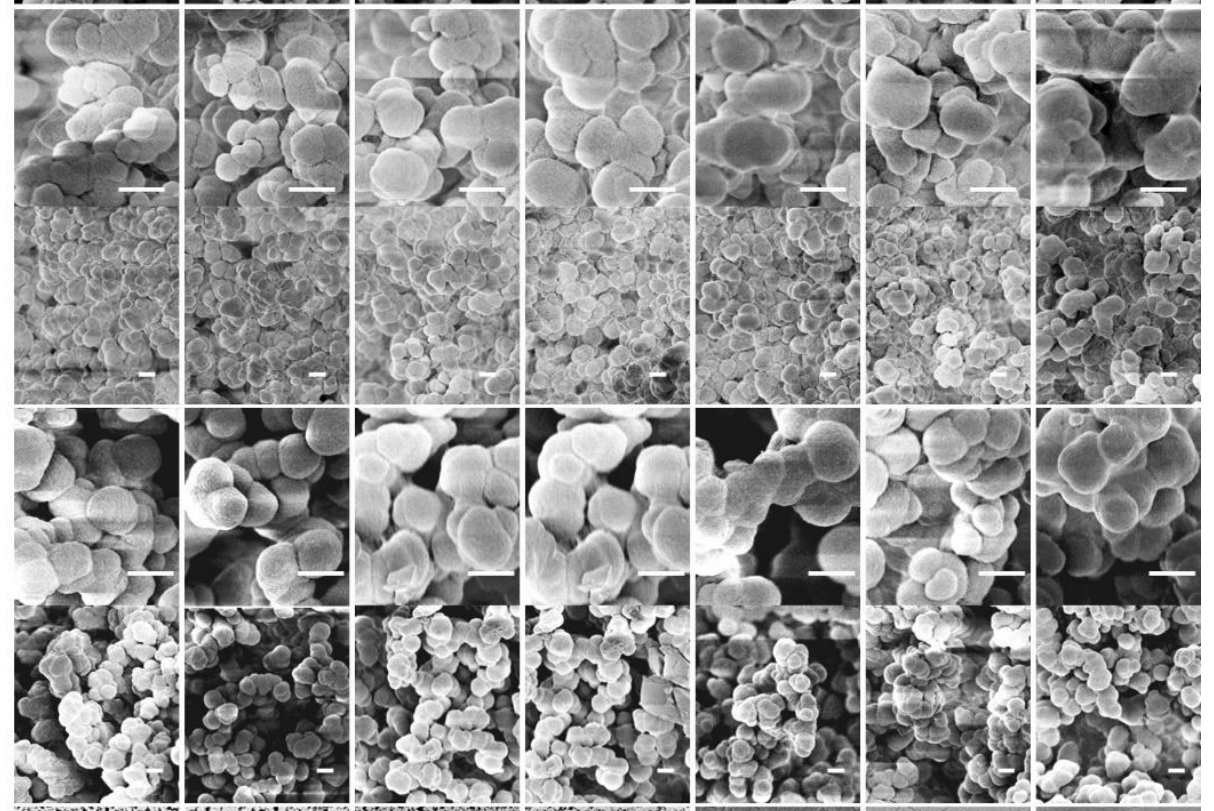

(D)

(E)
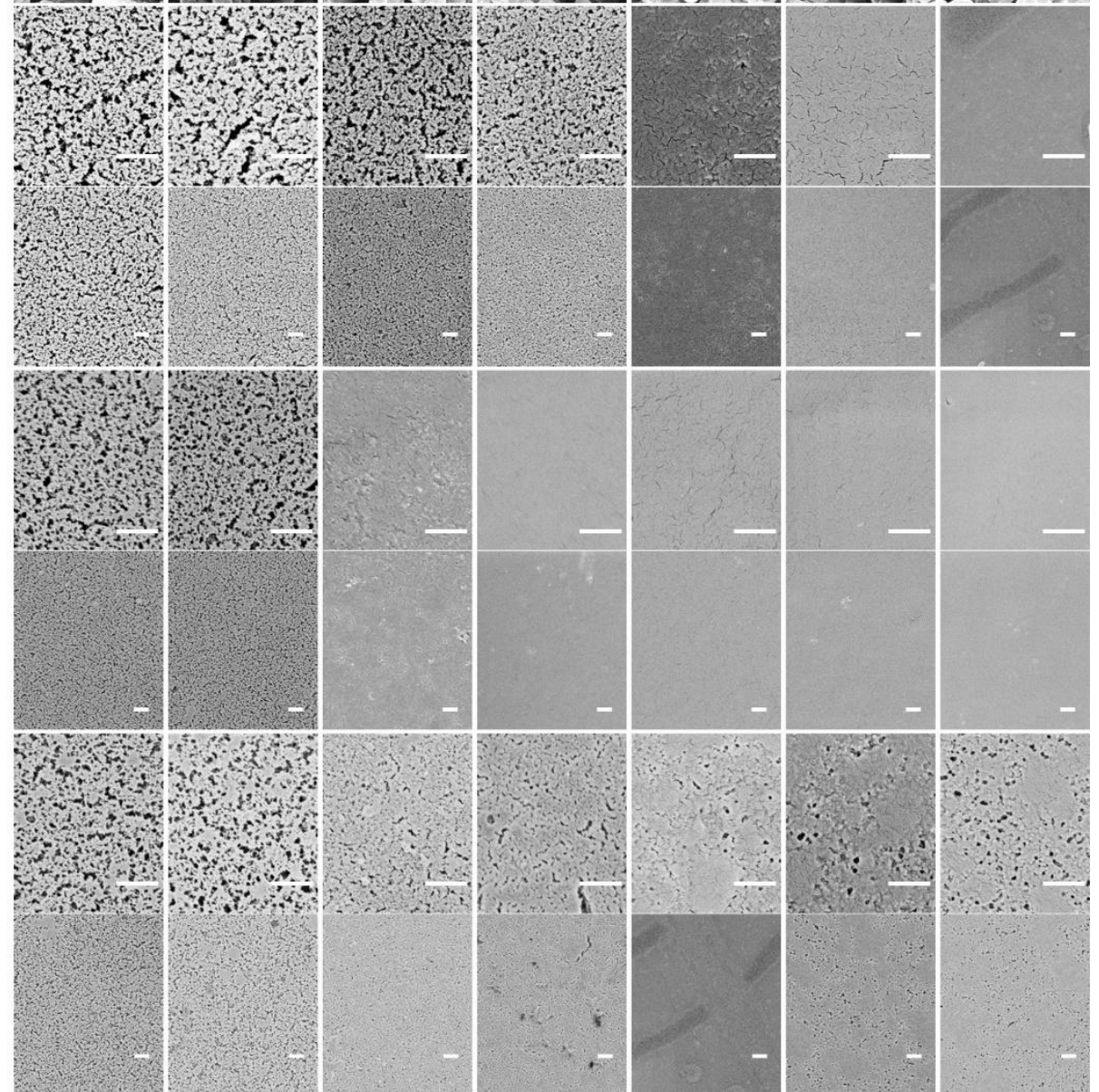

Figure S3. SEM images of (A)BM-AMPS surface, (B) BM-MDSA surface, (C) BM-META surface, (D) BN-AMPS surface, (E) BN-MDSA surface and (F) BN-META surface (scale bar: $1 \mu \mathrm{m}$ ). 


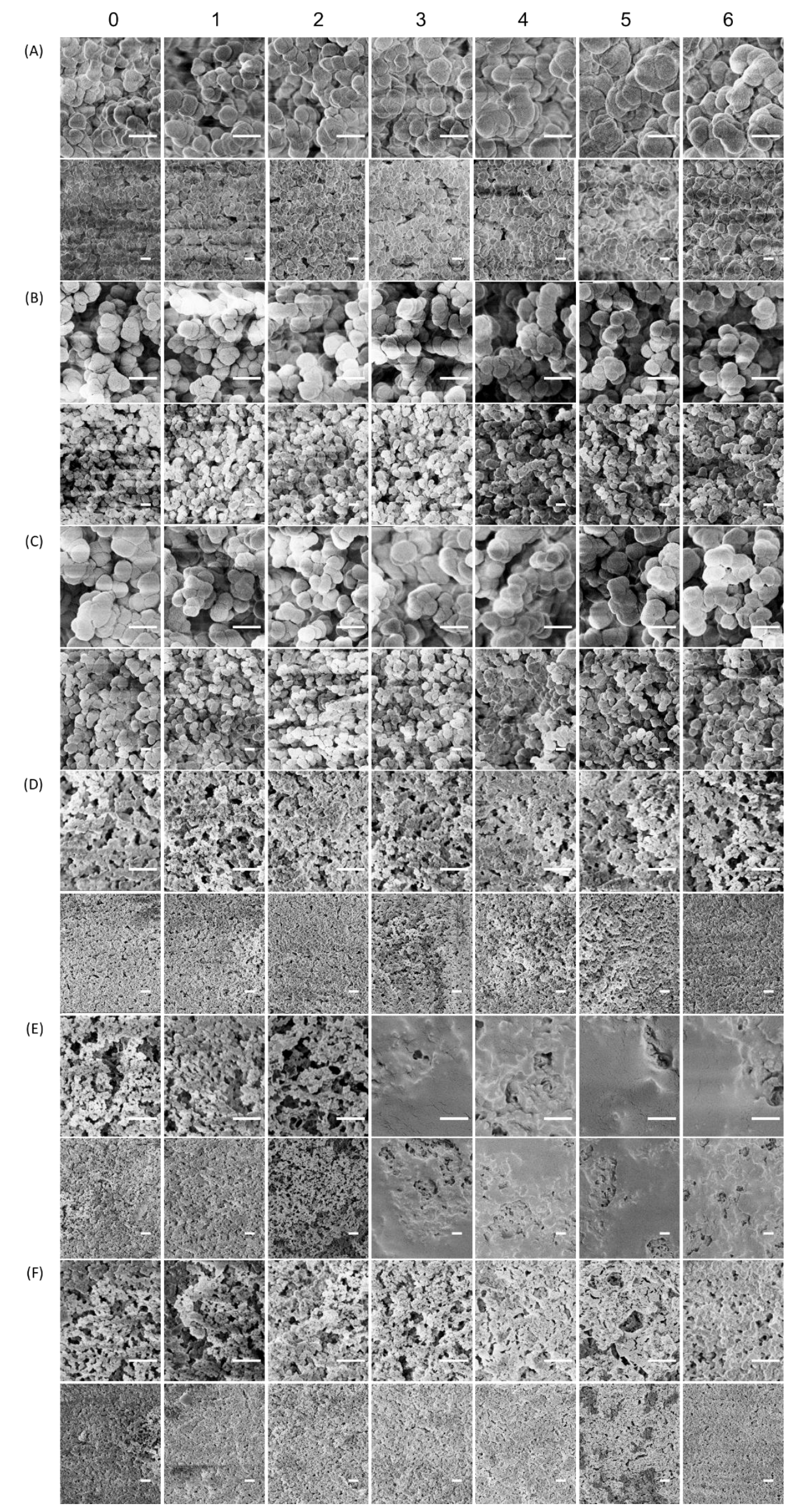

Figure S4. SEM images of (A)HM-AMPS surface, (B) HM-MDSA surface, (C) HM-META surface, (D) HN-AMPS surface, (E) HN-MDSA surface and (F) HN-META surface (scale bar: $1 \mu \mathrm{m}$ ) . 
A

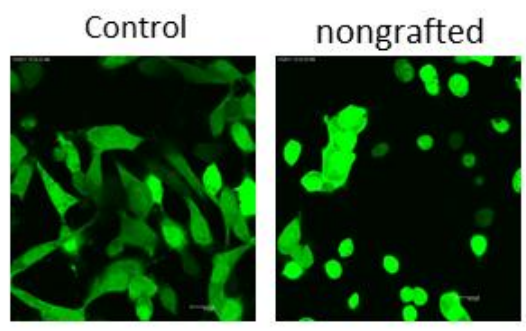

BM
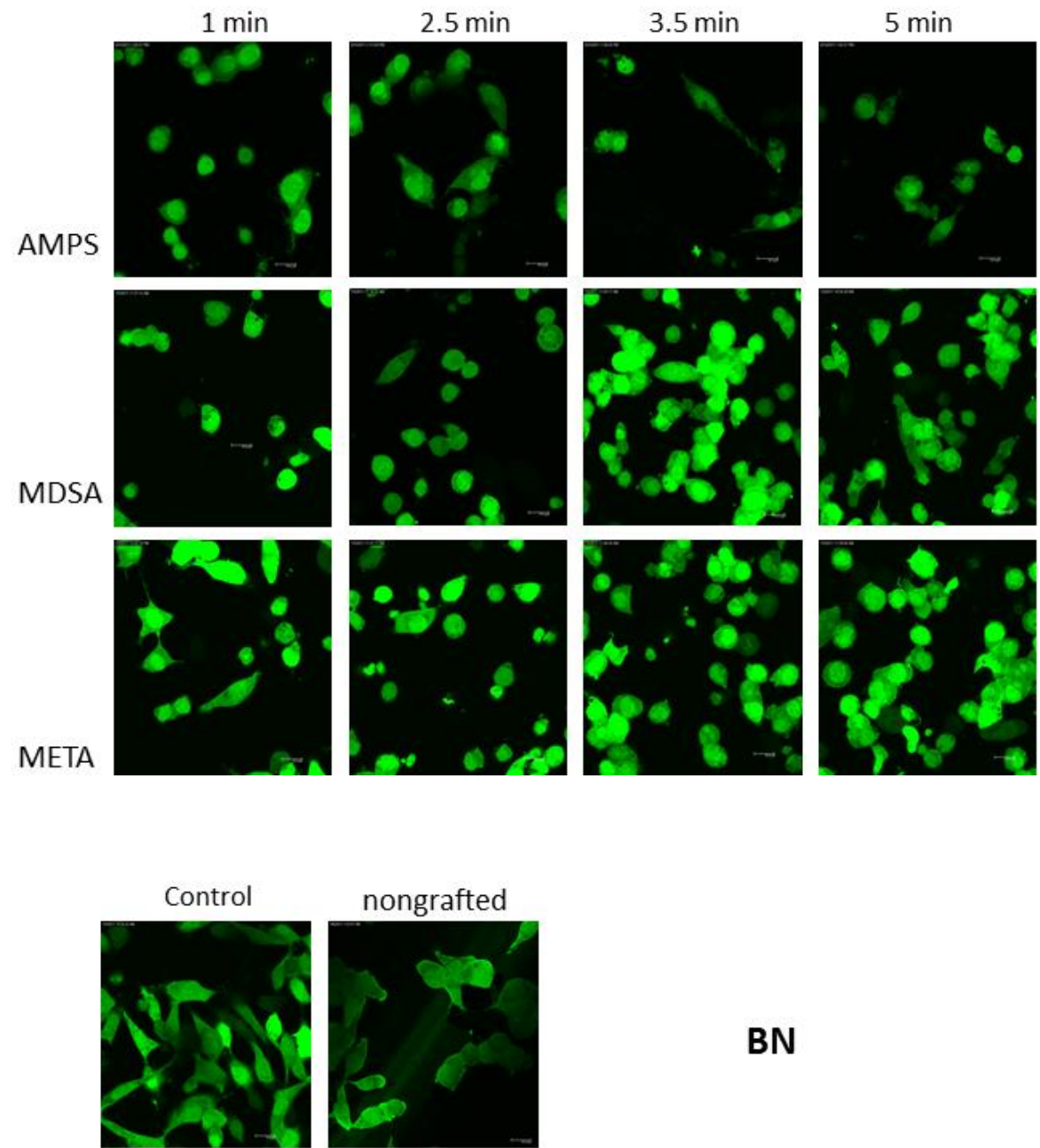

nongrafted

B

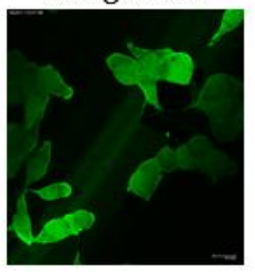

BN

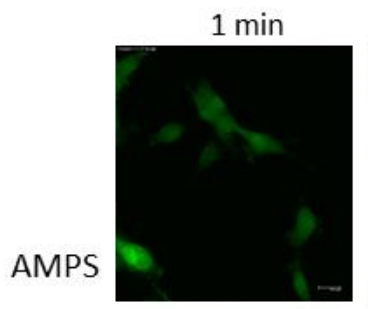

$2.5 \mathrm{~min}$

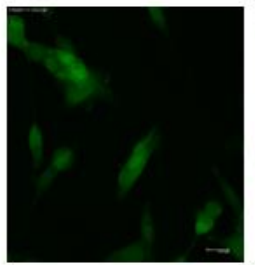

$3.5 \mathrm{~min}$
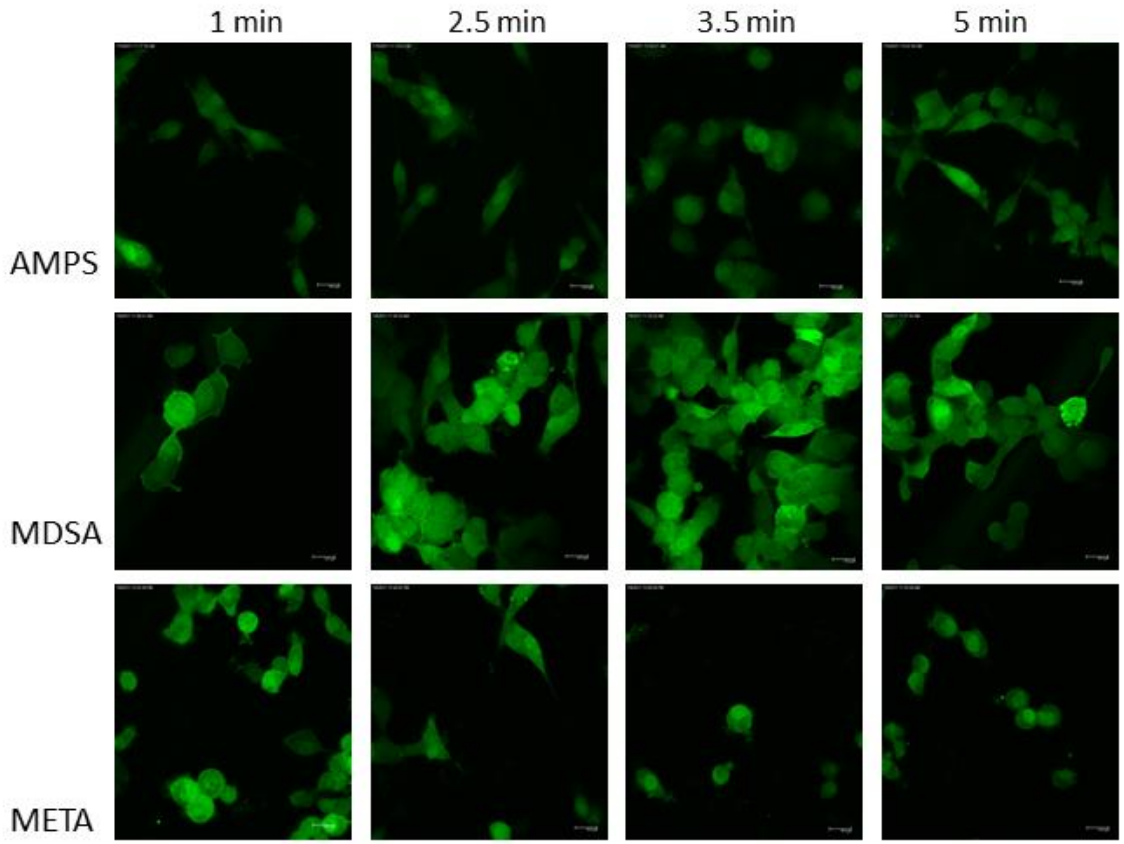


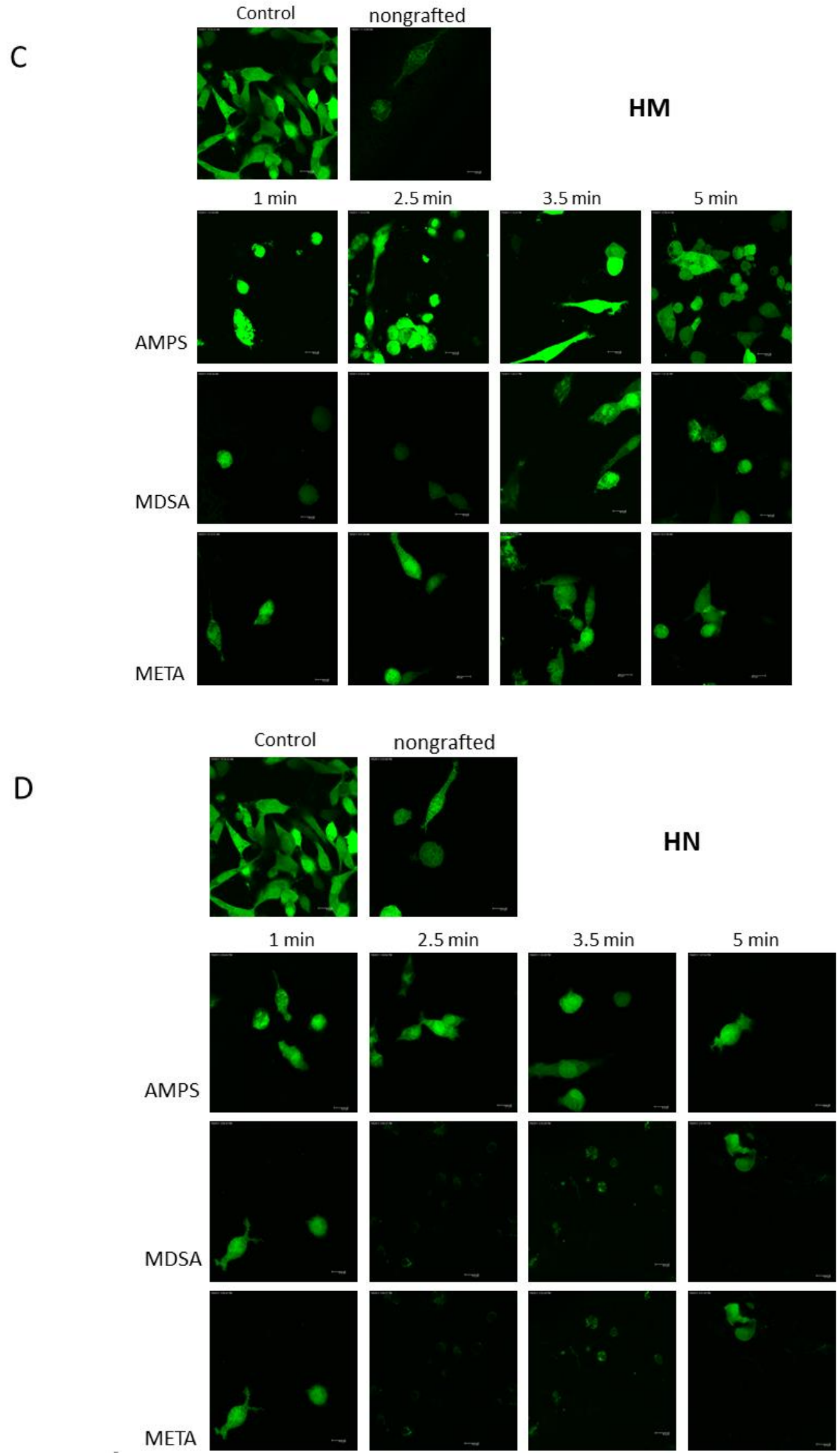

Figure S5. Representative images of the HT1080-GFP cells growing on grafted and non-grafted micro- and nano-porous surfaces (BM, BN, and HM, HN). In total 40 images from each glass slide were taken 24 hours after seeding. Cells were manually counted to estimate how different surfaces support cell growth.. 

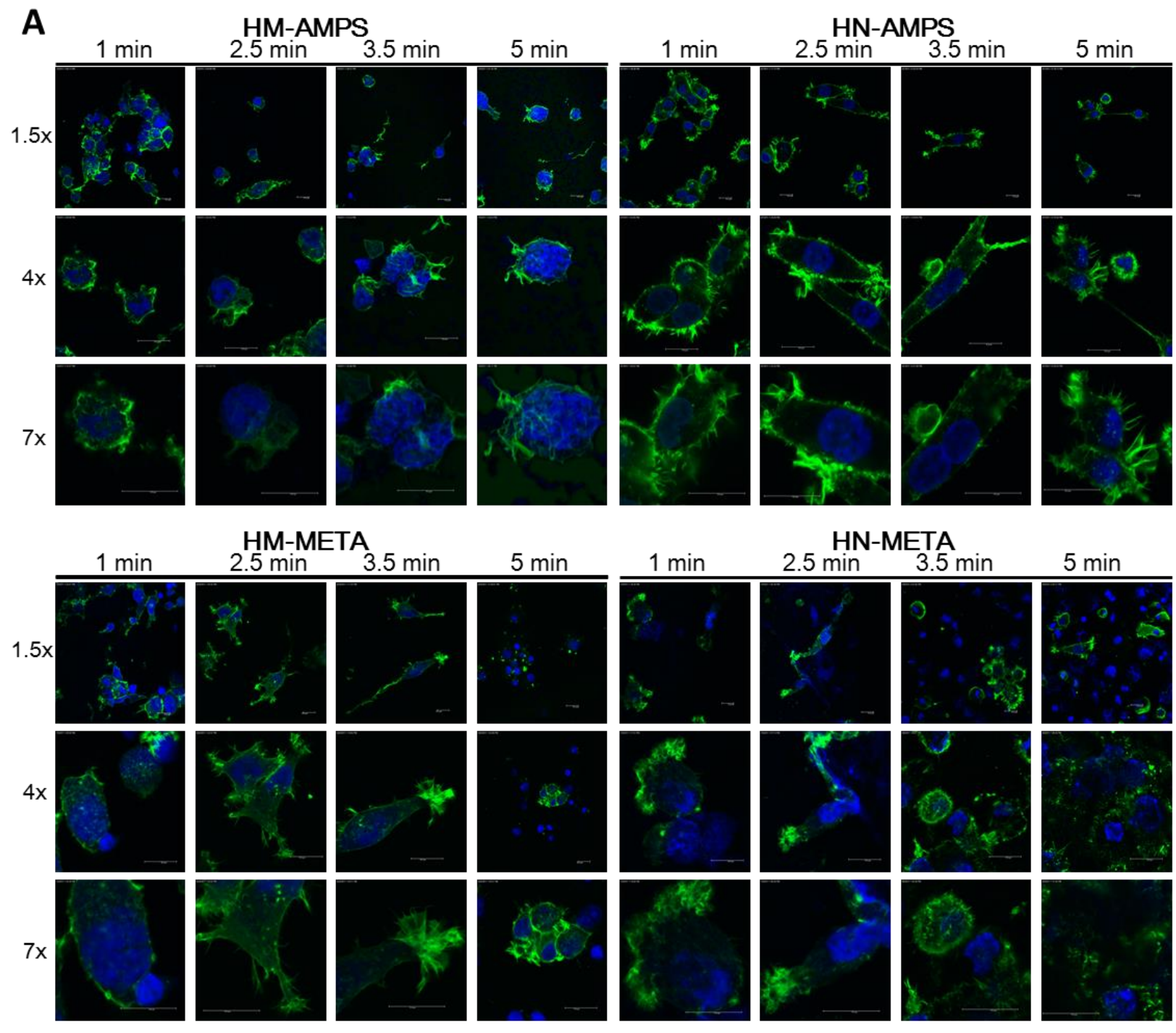

$5 \mathrm{~min}$
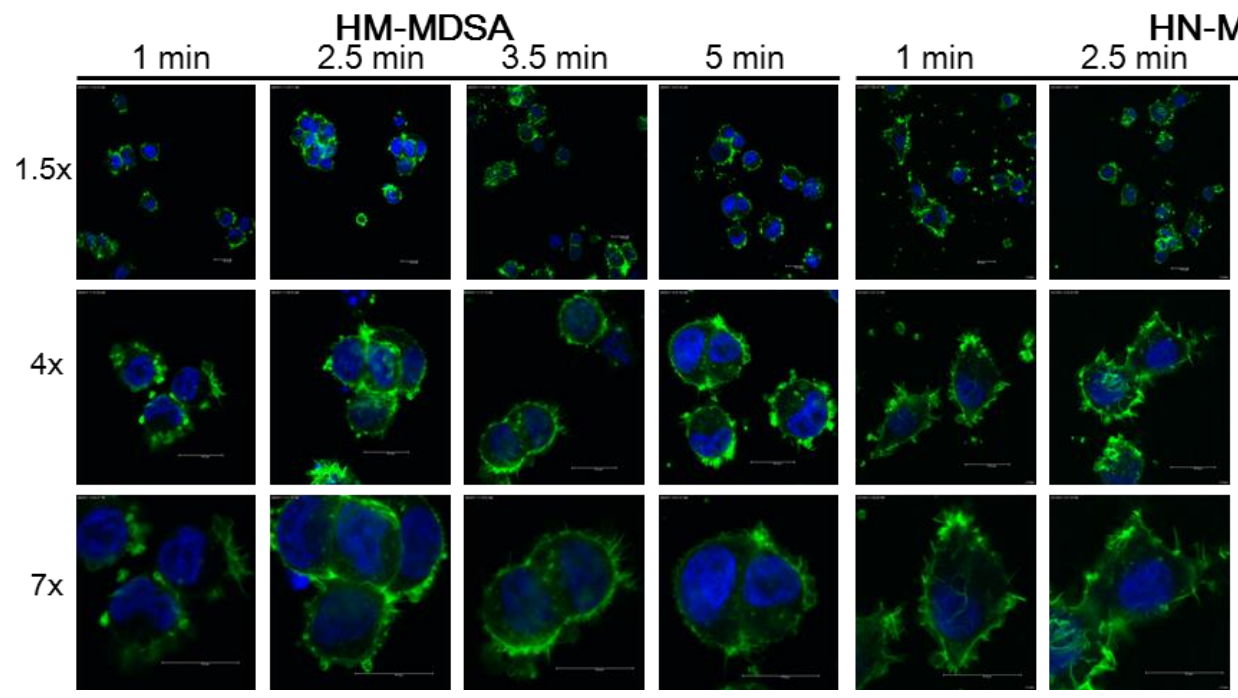

$5 \mathrm{~min}$

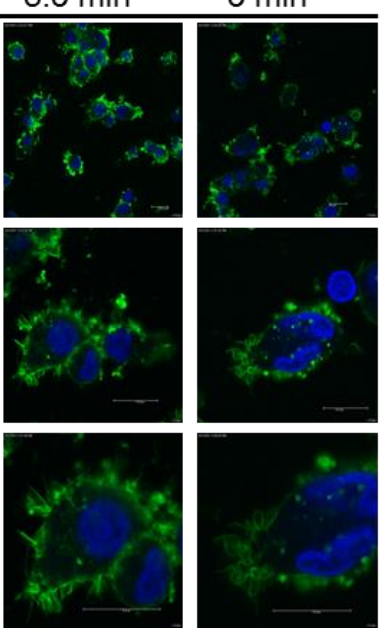




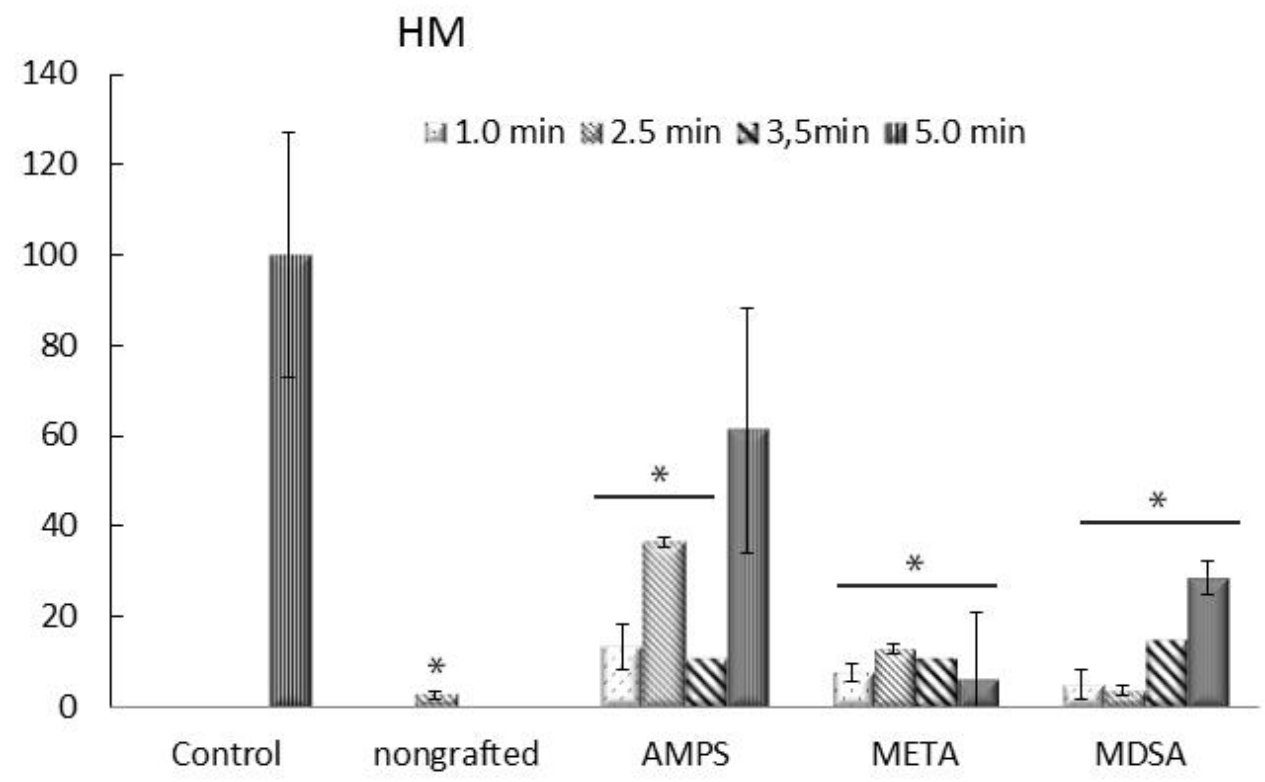

C

$\mathrm{HN}$

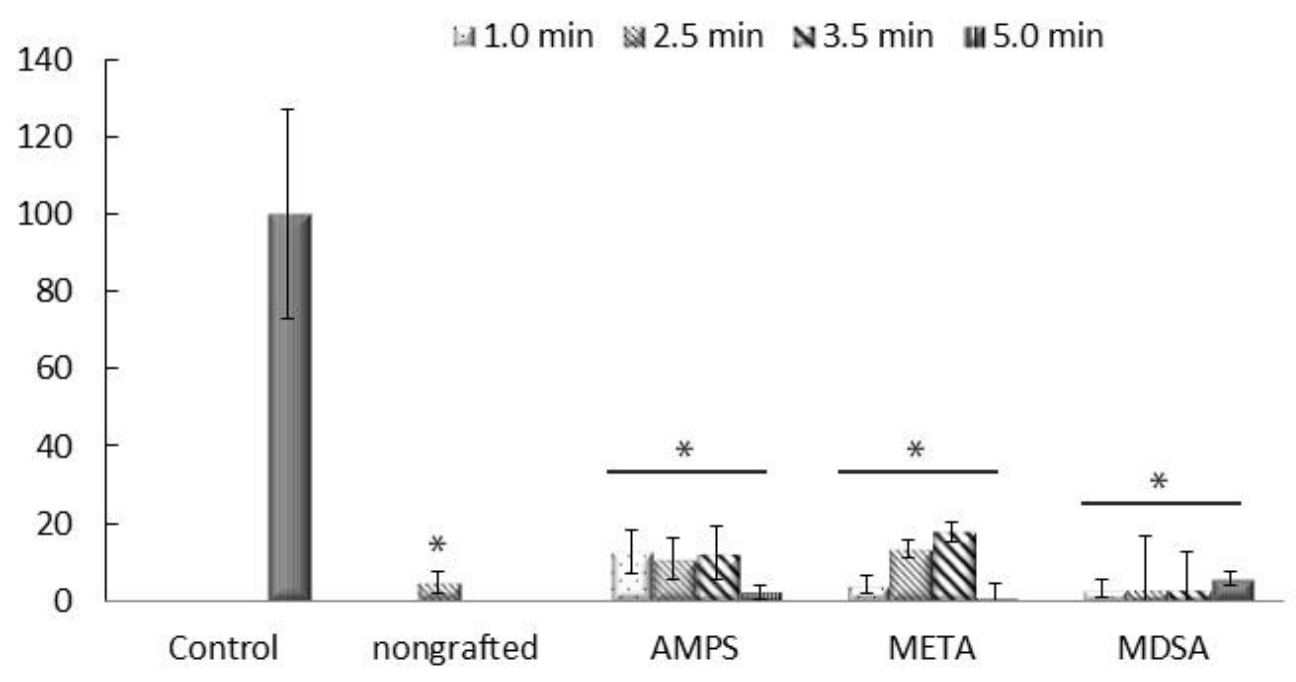

Figure S6 Analysis of cell morphology and growth on the HEMA-EDMA hydrophilic grafted surfaces.

A: Immunofluorescence images of HT1080 cells grown on the micro-porous HM-AMPS, HM-META, HM-MDSA surfaces, and on the nano-porous HN-AMPS, HN-META, HN-MDSA surfaces. The images were taken $24 \mathrm{~h}$ after seeding. A 1.5, 4 and $7 \mathrm{x}$ digital zoom was used in conjunction with a $63 \mathrm{x}$ water objective. Nuclei were stained with DAPI (blue) for visualization of cytoskeleton. F-actin was stained with phalloidin (green). Scale bar $15 \mu \mathrm{m}$. AMPS mediated cell aggregation on both, HM and HN surfaces, albeit cells exhibited more rounded shape on the HM surface indicating additional impact of surface topography (Fig. S6A, HM-AMPS, HN-AMPS). Cells growing on META-grafted surfaces did not reveal differences in 
shape as compared to the cells growing on the control umgrafted surface (Fig. 6SA. HM-META. HN-META; Fig. 4A, HM, HN). MDSA grafting lead to cell aggregation, cells exhibited rounded shapes similar to those observed on the BM-MDSA and BN-MDSA surfaces (Fig. 6SA, HM-MDSA, HN-MDSA; BM-MDSA, BN-MDSA). $B, C$ : To analyze cell growth, HT1080-GFP cells were plated on the grafted HM surfaces $(B)$ and on grafted $\mathrm{HN}$ surfaces $(C)$. Cells were incubated for $24 \mathrm{~h}$ and the number of cells then counted. Significance was calculated compared to the cells growing on the control glass slide. 